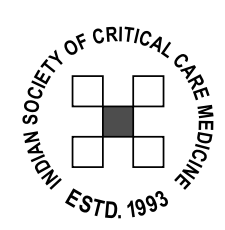

\title{
Guidelines for the Prevention of Infections Associated with the Use of Vascular Catheters in Indian Intensive Care Units Executive Summary of Recommendations
}

Committee for the Development of Guidelines for the Prevention of Vascular Catheter Associated Infection; Indian Society of Critical Care Medicine.

Members of the committee: Rajagopalan RE, (Chair); Arunkumar AS, Bhagwati AM, Divatia JV, Gopalakrishnan R, Kamat VN, Mani RK, Nagaraja P, Prayag S, Ramachandran B, Ramakrishnan N, Singhi S and Todi SK

\section{Introduction}

Blood stream infections associated with the use of vascular catheters carry significant morbidity. Increases in mortality attributable to such catheters have been clearly demonstrated. ${ }^{1}$ With greater use of these catheters in India, especially in the intensive care setting, there is an urgent need to develop infectionprevention guidelines that are sensitive to the local environment and practice patterns. While guidelines for prevention have been published in the West $^{2,3}$ their extrapolation, in an unmodified form, to the Indian milieu may not be appropriate. This executive summary presents India-specific recommendations made by an expert committee of the Indian Society of Critical Care Medicine (ISCCM). The major goal of the committee was not to develop Indian strategies de-novo, but to utilise pre-existing guidelines as the base, and to modify them based on Indian data and the opinions of the experts to provide a coherent set of recommendations that can be easy to apply in this country.

Besides catheter infection, many other clinical and practical concerns influence the choice of site, duration and care of vascular catheters. As a consequence, during the placement and use of vascular catheters, the recommendations of this committee should be weighed against these non-infectious considerations as well.

\section{Methods}

The ISCCM constituted an expert committee of intensivists, infectious disease specialists and microbiologists in August 2003. Subcommittees dealing with the specifics of the epidemiology of catheter infection, peripheral vascular catheters, central venous catheters, arterial lines and problems related to paediatric patients were given a four month period to review the guidelines of the American Centers for Disease Control and Prevention (CDC) ${ }^{2}$ and that of the UK Infection Control Society (UKICS). ${ }^{3}$ They also reviewed any recent literature that was published after the release of these guidelines and focussed on literature originating from India. The committee convened a 2-day consensus conference in December 2003, when the literature was presented and evidence was graded using a standardised method. ${ }^{4}$ Recommendations were graded into three categories based on the quality of evidence and on their interpretation by the expert committee (Table 1). The current paper provides only the final recommendations of the committee and references are provided only for features that differ from other published guidelines. Full publication of the evidence-base and rationale is planned for a later date.

Table 1: Modified Infectious Diseases Society of America-United States Public Health Service Grading System for used for ranking recommendations in this executive summary 4

\section{Quality of evidence}

Grade

I Evidence from $\geq 1$ properly randomized, controlled trial

II Evidence from $\geq 1$ well-designed clinical trial, without randomization; from cohort or case-controlled analytic studies (preferably from $\geq 1$ center);

from multiple time-series; or

from dramatic results from uncontrolled experiments

III Evidence from opinions of respected authorities, based on clinical experience,

descriptive studies, or reports of expert committees

\section{Strength of recommendation}

Category

A Good evidence to support a recommendation for or against use

B Moderate evidence to support a recommendation for or againstuse

C Poor evidence to support a recommendation 


\section{SECTION 1}

\section{Epidemiology}

While the epidemiology of catheter infections is well described in western literature, ${ }^{2}$ data from Indian hospitals is scarce. ${ }^{5-7}$ Though one study of intermediate-term central catheters in children reports rates of catheter associated bloodstream infection (CABSI; See appendix for definitions) of 1.3/1000 catheter days ${ }^{5}$ (unusually low even in comparison with Western data), anecdotally, rates are felt to be much higher in most Indian ICUs. The definitions of catheter infections are very variable in the published studies and need to be standardised. The committee was concerned about the high frequency of gram-negative infections in these reports, $, 6,7$ as it implies major inadequacies in site care, in the prevention of hub infections or the contamination of the infusate, ${ }^{8}$ medications administered and antiseptics used for site care. The high frequency of fungal colonisation of catheter tips and candidemia (about $45 \%$ ) in a paediatric study ${ }^{6}$ is also unusual and requires further evaluation.

\section{Recommendations}

1. All Institutions and intensive care units should measure CABSI rates $(A)$

2. For purposes of surveillance all BSIs count as CABSI (A)

3. Blood cultures are to be drawn only when BSI is clinically suspected (and preferably before antibiotics are started) (A)

4. Routine cultures of vascular catheter tips are not recommended $(A)$

Standard methodology for blood culture, vascular catheter-tip culture and calculation of CABSI rates are encouraged (Appendix).

\section{General measures to reduce risk of CABSI}

Though processes specific to catheter care are the most important way to reduce CABSI the committee emphasizes that broader measures aimed at training and education of healthcare workers would be equally important in the prophylaxis of these infections.

\section{Recommendations}

5. Healthcare workers need to be educated and trained in the insertion, maintenance and use of vascular catheters. Infection control programs should be developed and strictly adhered to $(A)$

6. Attempts should be made to guarantee adequate staffing to minimize $B S I(A)$

7. Dedicated vascular access teams are recommended when feasible $(A)$

8. Systemic antibiotics are not recommended for the prevention of CABSI (A)

9. All vascular catheters should be removed when there is no clinical indication for their continuation $(A)$

\section{Hand Hygiene 9}

As studies related to nosocomial infection clearly demonstrate a reduction in their occurrence with adherence to good hand hygiene, we feel that it should be incorporated into protocols of catheter care as well. Specific deviations from this general protocol are reported in each section.

\section{Recommendations}

10. Strict hand hygiene with alcohol based hand rubs or with liquid antiseptic soap and water is recommended before and after every contact with the patient (A)

11. Hand hygiene is mandatory even with the use of gloves $(A)$

A protocol for hand hygiene is provided in the appendix. 


\section{SECTION 2}

\section{Short peripheral intra-venous catheters}

Short, peripheral intravenous catheters (IV) are the most commonly used vascular access method in hospitals and intensive care units. Available data indicate that BSI rates are very low with the insertion of these catheters. On the other hand phlebitis induced by local infection, mechanical trauma and the corrosiveness of injectates and drugs is very common. The recommendations made in this section aim to reduce the frequency of phlebitis.

The committee has been sensitive to the cost issues associated with the frequent catheter changes that are usually recommended, ${ }^{2}$ and has suggested that, in the absence of local complications, peripheral i.v. catheters be left in-situ for longer durations of time, based on an analysis of current evidence $e^{10-15}$. In addition recent data clearly indicates that disinfecting the hands with an antimicrobial rub or wearing clean gloves during catheter insertion reduces the rate of local complications, including phlebitis, when compared with placing lines with bare hands after routine hand wash with a non-medicated soap. ${ }^{16}$ Finally, many local practices such as securing the catheter by direct application of an adhesive plaster (without sterile gauze) and discontinuous use of intravenous administration sets have not been adequately evaluated in the literature for the committee to make well-defined recommendations. The committee also felt that the use of venous cut-downs should be discouraged when percutaneous access options were available.

\section{Recommendations}

\section{Site preference}

1. In adults, IVs should be inserted in the hand rather than wrist or forearm (A)

2. Insertion of IVs in the lower extremity is not recommended except in children (B)

\section{Catheter material}

3. Use either non-PVC (polyvinyl chloride) plastic catheters or steel needles (e.g. scalp vein sets) to minimize the incidence of phlebitis. However, there is an increased potential for extravasation with steel needles $(A)$

\section{Hand hygiene ${ }^{16}$}

4. During catheter insertion, hand disinfection using an antiseptic rub is preferred to routine wash with unmedicated soap (A)

5. The use of clean gloves after hand hygiene is recommended (A)

\section{Skin preparation}

6. Prepare clean skin with $70 \%$ alcohol solution $(A)$

7. Use of acetone or ether is not recommended $(A)$

8. Once prepared, the site of insertion should not be palpated (A)

\section{Dressings; types \& frequency of change}

9. Use either sterile gauze or sterile transparent dressing at the catheter site $(A)$

10. No recommendation can be made on the practice of direct application of adhesive plaster to secure catheters (Unresolved)

11. Keep dressings on for the duration of the catheter, unless it is soiled, loosened or moist. (A)

\section{Monitoring \& Surveillance of site}

12. Daily palpation over the intact dressing for signs of phlebitis is essential $(A)$

13. Routine removal of gauze dressings is not recommended for surveillance of the site $(A)$

14. The dressing should be removed if palpation through the gauze is not possible, or if tenderness warmth or swelling is noted during surveillance. Direct inspection of the site is essential in these situations.

\section{Duration \& Catheter replacement ${ }^{10-15}$}

15. Do not routinely replace catheters before 96 hours unless there are signs of phlebitis, infection or malfunction (A)

16. In the absence of phlebitis, infection or malfunction, IVs may be left for longer periods with close surveillance of the site. (B)

17. In children, IVs may be left in place as long as clinically necessary, unless there are signs of phlebitis, infection or malfunction $(A)$

\section{Replacement of administration sets}

18. Do not routinely replace administration sets before 96 hours (B)

19.Administration sets used to infuse lipid based parenteral solutions and blood products should not be left in place for longer than 24 hours (A)

20. No recommendation can be made from existing data on the practice of reusing administration sets in a given patient for discontinuous infusions (Unresolved). However, the consensus group discourages this practice $(\mathrm{C})$

\section{Value of in-line filters}

21. There is no value of in-line filters for the prevention of catheter infection $(A)$ 


\section{Stopcocks \& side ports}

22. Catheters with injection side ports can be safely used with standard hygienic precautions (A)

23. Cap all stopcocks which are not being used (A)

24. Clean injection ports with $70 \%$ alcohol before access $(A)$

\section{Cut-downs}

25. Do not routinely use cut downs for peripheral venous access (A)
26. In an emergent situation when no alternative route of venous access is possible, a cut-down approach to peripheral venous access may be attempted. Such a catheter should be removed within 24 hours $(A)$

\section{Anticoagulant flush ${ }^{8}$}

27. In catheters at low risk for local complications, heparin is not recommended over normal saline flushes for the maintenance of patency or the reduction of phlebitis. (B) 


\section{SECTION 3}

\section{Central Venous Catheters}

\section{(With a contribution from Dr. A. Date)}

Central venous catheters (CVC) are now widely used in Indian intensive care units. They are used as vascular access for haemodynamic monitoring, parenteral nutrition, and the administration of fluids and drugs. However, these central venous catheters amplify the risk for local and systemic infections and increase the mortality rates of patients. Given that the predisposition for CRBSI is influenced by the duration and purpose of the central venous catheter, the committee has focussed only on short-term non-tunnelled catheters. Thus long-term catheters used for parenteral nutrition, haemodialysis and chemotherapy are not covered by these guidelines. Peripherally inserted central catheters are also not discussed.

Once again, the committee focussed on the costs involved in frequent replacement of the catheters and in the use of newly developed antibiotic- and antiseptic-impregnated catheters. With the published experience overwhelmingly against routine (scheduled) changes of central catheters all current guidelines recommend prolonged use of CVCs. ${ }^{2,3}$ The committee endorsed this opinion. However, though there is high quality data in favour of the impregnated catheters, ${ }^{18-21}$ the enormous expenses involved in the purchase of these catheters and the concerns about the inadequacies of older versions of these catheters, has led the committee to de-emphasize their role in the prevention of CRBSI and has shifted the focus to simpler, less expensive alternatives.

Thus the use of maximal sterile barriers during placement of catheters, ${ }^{22}$ the avoidance of the femoral site for catheter placement in adults ${ }^{23,24}$ and appropriate catheter site preparation prior to insertion were viewed as far more cost-effective processes in reducing catheter infection. With regards to the last issue, the committee felt that though the evidence in general favoured aqueous or alcohol-based preparations of chlorhexidine, ${ }^{25}$ there are some questions regarding the efficacy of preparations with lower concentrations of chlorhexidine. ${ }^{26}$ We have suggested that $10 \%$ povidone iodine solution is an acceptable alternative at present. We also noted that the vast majority of Indian formulations of povidone iodine are only of $5 \%$ strength and this concentration has not been evaluated in clinical studies of catheter infection. We recommend that the lower strength of this solution not be used in the preparation of the CVC site.

\section{Recommendations}

\section{Site preference}

1. In adults, subclavian vein cannulation is preferred to the jugular or femoral sites to reduce risk of infection. (A)

2. Any site (including the femoral vein) is acceptable in children as the infection risks are not significantly different $(B)^{24}$

3. In the selection of a site, weigh the non-infectious risks (haemorrhage, pneumothorax) against the benefits of reducing CRBSI $(A)$

\section{Catheter material \& type}

4. Use Polyurethane or Teflon ${ }^{\circledR}$ catheters rather than catheters made of polyvinyl chloride/polyethelene to reduce risk of infection (B)

5. The decision to use multi-lumen catheters and the number of lumens used should be influenced by the clinical needs of the patient, rather than by the marginally increased risk of infection $^{27}$ associated with use of multi-lumen catheters (B)

\section{Antibiotic coated Catheters}

6. Antibiotic coated catheters may be used for short term CVCs likely to remain in place for more than five days (A)

7. They may be considered in patients with high risk of infection (neutropenia, burns) (C)

8. The use of antibiotic coated catheters should not be a substitute for strict adherence to catheter insertion and maintenance protocols $(A)$.

\section{Hand hygiene}

9. Hand hygiene procedures must be strictly followed (even when gloves are worn) before and after injection, blood sampling, dressing or any contact with the CVC or insertion site. (A)

10. For the insertion of CVCs, full scrub (up to the elbows) with an antiseptic solution is recommended (A)

\section{Aseptic technique}

11. Use maximal sterile barrier precautions for the insertion of a central venous catheter. (A). These include the use of cap, mask, sterile gloves, full-sleeved sterile gown, and large sterile drapes.

12. Pulmonary artery catheters should have a sterile protection sleeve. (A)

\section{Skin preparation}

13. Aqueous or alcoholic chlorhexidine $(0.5-2 \%)$ is preferred to povidone-iodine for cleaning the skin prior to CVC insertion (A).

14. If chlorhexidine solutions are not available, $10 \%$ povidoneiodine is an acceptable alternative. (B)

$15.5 \%$ povidone-iodine solution is not recommended for site preparation (B) 
16. The antiseptic solution must be allowed to dry on the skin before insertion of the CVC. Povidine-iodine must remain on the insertion site for at least 2 minutes, if not dry before insertion. (A)

17. Do not apply organic solvents (e.g. ether) to the skin before insertion of the catheter or during dressing changes. (A)

\section{Dressings; types; frequency of change}

18. Use either sterile gauze or sterile, transparent, semipermeable dressing to cover the catheter site. (A)

19. Do not replace dressings daily. However any dressing that is moist, loosened, or soiled should be replaced (A).

20. A Gauze dressing must be replaced every 2 days, whereas transparent dressings must be changed every 7 days (A)

21. When there is excessive perspiration over the catheter insertion site, or if the site is bleeding or oozing, a gauze dressing is preferred over a transparent dressing. (C)

22. Do not use topical antibiotic ointments or creams at the insertion site $(A)$.

23. No recommendations are made regarding use of povidone iodine ointment at the insertion site (Unresolved issue) except for haemodialysis catheters $(A)$

\section{Monitoring \& Surveillance of site}

24. When gauze dressings are changed every 2 days, the site must be inspected for purulence and erythema and palpated for tenderness and induration $(A)$

25. In patients with transparent dressings, daily surveillance of the site should be performed without the removal of the dressing $(A)$

\section{Systemic antibiotics}

26. Administration of intra-nasal or systemic antibiotics to prevent catheter colonization or BSI is not recommended, either prior to catheter insertion or during catheter maintenance $(A)$

\section{Anticoagulant flush / lock}

27. Heparin flushes and locks used to prevent catheter thrombosis are not recommended for the prevention of infection $(A)$

\section{Catheter replacement}

28. Routine (timed) replacement of CVCs is not recommended as a method of infection-control (A).

29. Do not routinely replace catheters in patients with fever explained by another focus of infection or non-infectious cause (B)

30. When a CVC has been placed with less than maximal sterile precautions (e.g. in an emergency) it should be replaced as soon as possible, and in any case, no later than 48 hours after insertion (C)

31. Replace any short-term CVC if purulence is observed at the insertion site $(A)$

32. Replace all CVCs if the patient is hemodynamically unstable and CRBSI is suspected (B)

33. Remove catheters as soon as they are not needed. (A)

\section{Over-the-guide wire exchanges}

34. Routine replacement of CVCs over a guide wire is not recommended as an infection-control measure. (A)

35. A CVC may be changed over a guide wire to replace a malfunctioning catheter or to convert an existing catheter (e.g. CVC to pulmonary artery catheter or vice versa) if there is no evidence of infection at the catheter site. (B)

36. Maximal sterile barrier precautions must be used during an over-the-guide wire catheter exchange. After removal of the old catheter, a new set of sterile gloves must be used before handling the replacement catheter. $(B)$

37. If CABSI is suspected or documented, guide wire-assisted exchange should not be undertaken $(A)$. The old catheter must be removed and a new catheter inserted at a fresh site (A) 


\section{SECTION 4}

\section{ARTERIAL LINES}

The risk of colonization and infection of arterial catheters in general approach that of central venous catheters (internal jugular \& subclavian). In general, care of these catheters is identical to the maintenance of central venous catheters. Only aspects that are significantly at variance with the recommendations for CVCs are mentioned in this section.

\section{Recommendations}

\section{Site preference}

1. The preferred sites for insertion of arterial lines in adults are radial, dorsalis pedis \& femoral (B). Axillary artery lines carry a high risk of infection.

2. The posterior tibial artery is an additional site in children

\section{Hand hygiene ${ }^{28}$}

3. All arterial line insertions using the Seldinger technique and all femoral arterial catheterizations should be performed using maximal sterile barrier precautions (masks, cap, sterile fullsleeved gowns, sterile gloves, \& large sterile drapes) (A)

4. Placement of radial and dorsalis pedis arterial lines without a guide wire can be performed after hand hygiene using an antimicrobial soap / scrub and wearing of sterile gloves (B)

Skin preparation (see CVC recommendations)
Dressings; types; frequency of change (see CVC recommendations)

Monitoring \& Surveillance of Site (see CVC recommendations)

\section{Duration \& Catheter replacement}

5. Arterial catheters can safely be left in situ for 96 hours (A). Catheters may be left in place longer if clinically required with ongoing surveillance of the site(B).

Selection \& replacement of pressure monitoring systems

6. Use disposable rather than reusable transducer monitoring systems whenever possible (A)

7. Replace the entire disposable transducer system at 96 hour interval $(A)$

8. Replace re-usable transducer systems every 48 hour (B)

\section{Care of pressure monitoring systems}

9. Maintain patency using a closed continuous flush system rather than an open system to reduce risk of infection $(A)$.

10. Keep all components of the pressure monitoring system sterile (A)

11. Minimize the number of manipulations and entries into the pressure monitoring system (B)

12. Do not administer dextrose containing solutions through the pressure monitoring circuits $(A)$

13. Sterilize reusable transducers according to manufacturer's instruction if the use of disposable transducer is not feasible (A) 


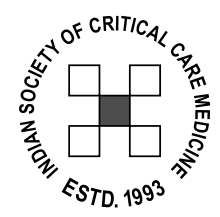

\section{APPENDIX}

\section{Blood and catheter culture methodology $29-32$}

\section{Blood Culture}

Several protocols are available for blood cultures; the following is suggested to maximize yield and minimize contamination rates. ${ }^{29,30}$

1. Blood cultures should be drawn only when there is a clinical suspicion of bloodstream infection.

2. Number: At least two sets of blood cultures must be drawn in each instance. Three sets may be needed to establish continuous bacteremia.

3. Site: Peripheral venepuncture is preferred for blood draws. Blood should not be drawn from catheters that are already in place. (If venous sites are unavailable, cultures may be drawn from freshly placed central vascular lines, observing strict aseptic precautions.) Each set should be drawn from a different venous site (to minimize false positives from site contamination).

4. Timing: The cultures should preferably be drawn prior to the administration of antibiotics. Though it is suggested that blood cultures should be drawn as soon as possible after an episode of fever or rigors, this does not affect yield significantly.

5. The timing between each set is undetermined and will depend on the urgency to treat the patient with antibiotics. (Cultures drawn 10-15 minutes apart are acceptable in the intensive care patient).

6. Hand care: Sterile gloves should be worn after hand cleansing with antimicrobial solution/ soap. The prepared site should not be palpated without gloves.

7. Site preparation: Tincture of iodine or $0.5 \%$ chlorhexidine in alcohol is preferred over povidone iodine for the preparation of the venepuncture site.

8. Volume of blood: Each set should not be less than $10 \mathrm{ml}$ in adults and should ideally be between 20 and $30 \mathrm{ml}$.

9. While injecting the blood into culture bottles the rubber stopper should be disinfected with alcohol. Do not change needles to transfer contents to the culture bottle.

\section{Catheter Culture ${ }^{31,32}$}

Multiple qualitative, semiquantitative and quantitative methods are available for identification of catheter colonization. In general, though quantitative methods perform best in the diagnosis of catheter related infection, ${ }^{32}$ the widespread unavailability of the technique restricts its value in India. We suggest that standardized reporting of short-term catheter related infections in India utilize the semiquantitative method of Maki ${ }^{31}$ described below:

1. Catheters removed on suspicion of catheter related bloodstream infection should be cultured. Routine culture of all removed vascular catheters is not recommended.

2. The catheters should be removed using sterile techniques (sterile gloves after appropriate hand disinfection).

3. The catheter site is cleared of any blood or antimicrobial ointment using an alcohol pledget.

4. The catheter is withdrawn with sterile forceps, directing it away from the skin.

5. If the catheter is $<7 \mathrm{cms}$ long the entire catheter is cultured. For longer catheters, a 5-7 cm segment either from the tip or from the skin-catheter interface is used for the culture.

6. The segment is rolled or smeared at least 4 times over a blood agar plate using a flamed forceps.

7. The number of colonies grown on the plate is counted. Counts exceeding 15-colonies/ plate or the presence of confluent growth is considered significant.

\section{Definitions $^{2}$}

Bloodstream infections (BSI) are identified by the growth of pathogenic bacteria or fungi (that are not related to another site of infection) from one or more blood cultures. When the blood culture yields a potential skin contaminant (Coagulase negative Staphylococcus, diphtheroids or Bacillus spp.) more stringent criteria are needed. In such cases the presence of more than one positive culture, and the presence of systemic signs and symptoms (fever, chills and hypotension) and the absence of an alternative focus of infection are essential.

Catheter associated bloodstream infections (CABSI) are those that occur in patients during and up to 48 hours after the removal of a central venous access or arterial catheter. This does not require the growth of the same organism from both the catheter and the peripheral blood.

Catheter-related bloodstream infections (CRBSI) require the isolation of the same organism in the blood culture and from the catheter site. Quantitative or semi-quantitative culture methods are required to differentiate catheter colonization from contamination of the site.

\section{Calculation of CABSI rate}

We recommend that all users of central catheters document the 
"incidence-density" of catheter associated blood stream infections in their institution. This is done looking at the frequency of BSI in all patients who had a catheter in place during or up to 48 hours prior to the positive blood culture. The incidence-density is obtained by calculating the number of BSIs per 1000 catheter days:

[No. of blood stream infections/ No. of catheter-days] x 1000;

where "catheter-days" = total number of days on a central venous or arterial catheter.

\section{A Protocol for hand hygiene ${ }^{33,9}$}

\section{Routine Hand Hygiene}

a. This process is mandatory for all medical, nursing and paramedical personnel who come in contact with the patient.

b. All such individuals should wash their hands and distal forearm with water and $2 \%$ chlorhexidine surgical scrub solution at the beginning of their duty hours and after every break away from the unit.

c. No watches or jewellery must be worn during this wash. This wash must last for a minimum of two minutes.

d. The hands must be dried with a sterile, dry towel or with disposable paper tissue.

e. The process must be repeated after any accidental unprotected contact with the body fluids of a patient.

f. A hand rub (with alcohol or chlorhexidine in alcohol) should subsequently be used:
- Before and after every contact with the patient,

- While moving from one bed to another

- While moving to common areas (refrigerator/store/ telephone).

g. The hand rub is also required before gloving and after degloving for any non-procedural contact that involves handling of IV lines or fluids, blood draws or contact with body fluids or wounds.

h. The use of gloves does not preclude the need for the hand hygiene techniques described above.

\section{Procedural Hand Hygeine:}

a. This process is indicated before every procedure including, but not limited to, central venous access, PA catheter and arterial line placement.

b. The process is a full surgical scrub using running water and $4 \%$ chlorhexidine scrub solution from the fingertips to the elbow.

c. Care must be taken to ensure drainage of water away from the fingers and hands, towards the elbows.

d. No watches, rings or jewellery must be worn during the scrub.

e. There is no advantage to the use of sterile disposable brushes or sponges in the process. Reusable brushes or sponges should not be used.

f. The scrub should be performed for a minimum of two to three minutes.

g. Hand drying should be only with the use of sterile (autoclaved) towels.

h. This scrub always precedes gowning and gloving for the procedure. 


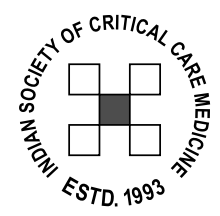

\section{REFERENCES}

1. Renaud B, Brun-Buisson C, for the ICU-Bacteremia Study Group. Outcomes of primary and catheter-related bacteremia: A Cohort and Case-Control Study in Critically III Patients. Am J Respir Crit Care Med 2001; 163: 1584-90.

2. O'Grady NP, Alexander M, Dellinger EP, Gerberding JL, Heard So, Maki DG, Masur H et al. Centers for Disease Control and Prevention. Guidelines for the Prevention of Intravascular Catheter-Related Infections. MMWR. 2002; 51(No.RR-10): 1-36

3. Guidelines for preventing infections associated with the insertion and maintenance of central venous catheters. J Hosp Infect. 2001; 47(Supplement): S47-S67

4. Gross PA, Barrett TL, Dellinger P, et al. Purpose of quality standards for infectious diseases. Infectious Diseases Society of America. Clin Infect Dis. 1994; 18:421.

5. Rao S, Alladi A and Cruz AJD. Medium and long-term central venous access in children. Indian Pediatrics. 2003; 40: 4144

6. Sachdev A, Gupta D, Soni A and Chugh K. Central venous catheter colonization and related bacteremia in Pediatric Intensive Care Unit. Indian Pediatrics. 2002; 39: 752-760

7. Verghese SL, Padmaja P, Koshi G.Central venous catheter related infections in a tertiary care hospital. J Assoc Physicians India 1998; 46: 445-7.

8. Lalitha MK, Kenneth J, Jana AK, Jesudason MV, Kuruvilla KA, Jacobson K, Kuhn I, Kronvall G. Identification of an IVdextrose solution as the source of an outbreak of Klebsiella pneumoniae sepsis in a newborn nursery. J Hosp Infect. 1999; 43: 70-3.

9. Boyce JM, Pittet D. Centers for Disease Control and Prevention. Guideline for Hand Hygiene in Health-Care Settings: Recommendations of the Healthcare Infection Control Practices Advisory Committee and the HICPAC/ SHEA/ APIC/IDSA Hand Hygiene Task Force. MMWR. 2002; 51(No. RR-16): 1-56

10. Lai KK. Safety of prolonging peripheral cannula and i.v. tubing use from 72 hours to 96 hours. Am J Infect Control. 1998; 26: 66-70.

11.Catney MR, Hillis S, Wakefield B, Simpson L, Domino L, Keller S, Connelly T, White M, Price D, Wagner K Relationship between peripheral intravenous catheter dwell time and the development of phlebitis and infiltration. J Infus Nurs. 2001; 24: 332-41.

12. Homer LD, Holmes KR. Risks associated with 72- and 96hour peripheral intravenous catheter dwell times. J Intraven Nurs. 1998; 21: 301-5.

13. Tager IB, Ginsberg MB, Ellis SE, Walsh NE, Dupont I, Simchen $E$, Faich GA. An epidemiologic study of the risks associated with peripheral intravenous catheters. Am J Epidemiol. 1983; 118: 839-51.
14. Bregenzer T, Conen D, Sakmann P, Widmer AF.Is routine replacement of peripheral intravenous catheters necessary? Arch Intern Med. 1998; 158: 151-6.

15. Garland JS, Dunne WM Jr, Havens P, Hintermeyer M, Bozzette MA, Wincek J, Bromberger T, Seavers M. Peripheral intravenous catheter complications in critically ill children: a prospective study. Pediatrics. 1992; 89:1145-50.

16. Hirschmann H, Fux L, Podusel J, Schindler K, Kundi M, Rotter $M$, Wewalka $G$; The influence of hand hygiene prior to insertion of peripheral venous catheters on the frequency of complications. EURIDIKI. European Interdisciplinary Committee for Infection Prophylaxis. J Hosp Infect. 2001; 49: 199-203.

17. Niesen KM, Harris DY, Parkin LS, Henn LT. The effects of heparin versus normal saline for maintenance of peripheral intravenous locks in pregnant women. J Obstet Gynecol Neonatal Nurs. 2003; 32: 503-8.

18. Veenstra DL, Saint S, Saha S, Lumley T, Sullivan SD. Efficacy of antiseptic-impregnated central venous catheters in preventing catheter-related bloodstream infection: a metaanalysis. JAMA. 1999; 281: 261-7.

19.Darouiche RO, Raad II, Heard SO, Thornby JI, Wenker OC, Gabrielli A, Berg J, et al. A comparison of two antimicrobialimpregnated central venous catheters. Catheter Study Group. N Engl J Med. 1999; 340: 1-8.

20. Veenstra DL, Saint S, Sullivan SD. Cost-effectiveness of antiseptic-impregnated central venous catheters for the prevention of catheter-related bloodstream infection. JAMA. 1999; 282: 554-60.

21. Marin MG, Lee JC, Skurnick JH. Prevention of nosocomial bloodstream infections: Effectiveness of antimicrobialimpregnated and heparin-bonded central venous catheters.Crit Care Med. 2000; 28: 3332-38.

22. Sherertz RJ, Ely EW, Westbrook DM, Gledhill KS, Streed SA, Kiger B, Flynn L, et al. Education of physicians-in-training can decrease the risk for vascular catheter infection. Ann Intern Med. 2000; 132: 641-8

23. Merrer J, De Jonghe B, Golliot F, Lefrant JY, Raffy B, Barre E, Rigaud JP, et al. French Catheter Study Group in Intensive Care. Complications of femoral and subclavian venous catheterization in critically ill patients: a randomized controlled trial. JAMA. 2001; 286: 700-7.

24. Venkataraman ST, Thompson AE, Orr RA. Femoral vascular catheterization in critically ill infants and children. Clin Pediatr (Phila). 1997; 36: 311-9.

25. Chaiyakunapruk N, Veenstra DL, Lipsky BA, and Saint S, Chlorhexidine Compared with Povidone-lodine Solution for Vascular Catheter-Site Care: A Meta-Analysis. Ann Intern Med. 2002; 136: 792-801.

26. Humar A, Ostromecki A, Direnfeld J, Marshall JC, Lazar N, 
Houston PC, Boiteau P, Conly JM. Prospective randomized trial of $10 \%$ povidone-iodine versus $0.5 \%$ tincture of chlorhexidine as cutaneous antisepsis for prevention of central venous catheter infection. Clin Infect Dis. 2000; 31:10017.

27. Dezfulian C, Lavelle J, Nallamothu BK, Kaufman SR, Saint $S$. Rates of infection for single-lumen versus multilumen central venous catheters: A meta-analysis. Crit Care Med. 2003; 31: 2385-90.

28. Rijnders BJ, Van Wijngaerden E, Wilmer A, Peetermans WE. Use of full sterile barrier precautions during insertion of arterial catheters: a randomized trial. Clin Infect Dis. 2003; 36: 7438.

29. Shafazand S, Weinacker AB. Blood cultures in the critical care unit: Improving utilization and yield. Chest 2002; 122:1727-36.

30. Aronson MD, Bor DH. Blood cultures. Ann Intern Med. 1987: $106 ; 246-53$.

31. Maki DG, Weise CE, Sarafin HW. A semiquantitative culture method for identifying intravenous-catheter-related infection. N Engl J Med. 1977; 296: 1305-9.

32. Siegman-Igra Y, Anglim, A, Shapiro DE, Adal KA, Strain BA, Farr BM. Diagnosis of vascular catheter-related bloodstream infection: a meta-analysis J Clin Microbiol.1997; 35: 92836.

33. Ridgway GL. Hygiene. Chapter 13.2.1 in Oxford Textbook of Critical Care; Webb AR, Shapiro MJ, Singer M, Suter PM, (Editors). Oxford University Press (Pub). 1999; 872-4. 


\section{ACKNOWLEDGEMENTS}

We are grateful for the support of $3 \mathrm{M}$ India Limited, Bangalore and especially Dr. Nirmala Balwalli, Technical Manager and Mr. Rajeev Menon, Marketing Manager, 3M India Limited, Bangalore. We thank Ms. Joanne P. Kubala, RD, LD, Clinical Educator, 3M Medical Division, USA, for reviewing the transcript and making valuable suggestions.
3M India Limited, Bangalore provided logistical support, access to published literature \& library resources and organized the two meetings convened in Chennai in 2003. The content of this executive summary was developed solely by the ISCCM expert committee. 